\title{
Use of the Kolsky method for dynamic tests of brittle media
}

\author{
Anatoliy Bragov ${ }^{1, *}$, Leonid Igumnov ${ }^{1}$, Andrey Lomunov ${ }^{1}$, Alexander Konstantinov ${ }^{1}$, \\ Dmitriy Lamzin ${ }^{1}$, and Leopold Kruszka ${ }^{2}$ \\ ${ }^{1}$ Research Institute for Mechanics, National Research Lobachevsky State University of Nizhni \\ Novgorod, Gagarin Ave., 23, bldg. 6, 603600, Russian Federation \\ ${ }^{2}$ MilitaryUniversity of Technology, 2 Gen. Witolda Urbanowicza Street, 00-908 Warsaw, Poland
}

\begin{abstract}
The dynamic test techniques used to obtain the mechanical properties of brittle materials are described. The techniques are based on the fundamental Kolsky method using the Split-Hopkinson Pressure Bar. Dynamic tests are characterized by high intensity and short duration and the influence of inertia on their results should be ruled out which is especially important for brittle media. The prerequisites and assumptions presented methods are described to justify the validity of the obtained data. The methods allow to obtain dynamic deformation diagrams at compression, splitting, indirect tension, shear and triaxial stress state and also to determine the ultimate strength, strain and time properties of brittle materials. The techniques are approved in the tests of ceramic bricks, finegrain concrete and fiber-reinforced concrete.
\end{abstract}

\section{Introduction}

In recent years, the study of the processes of dynamic deformation and destruction of structures is becoming particularly relevant. Dynamic effects are very diverse and can have both operational (for example, equipment loading, etc.) and extreme (for example, explosion, earthquake, etc.) character. Such effects are characterized by a continuous change in parameters, high intensity, short duration and sometimes limited action zone. In addition, innovative materials exhibit sometimes an unpredictable response to an applied dynamic loading.

The calculation of the stress-strain state of structures on dynamic effects has recently been carried out using finite element programs such as "Dynamics-2", ANSYS, LS-DYNA, ABAQUS, etc., where a variety of mathematical models of materials taking into account various factors are used. To provide them with the necessary parameters and constants, testing of materials is required. The dynamic properties in a wide range of strain rates are mainly obtained by the Kolsky method using the Split-Hopkinson Pressure Bar (SHPB) and its numerous modifications. To identify and verify complex models of deformation of materials, that consider deeper dynamic effects, modernization of existing test benches is required, as well as the development of new techniques for dynamic experiments. The study

* Corresponding author: bragov@mech.unn.ru 
in the outlined field does not stand still, that is proved by a large number of papers on this topic, for example [1-8].

The present paper is of a methodical character and devoted to the description of the Kolsky method and some of its modifications for testing brittle media, as well as the main assumptions underlying them. Features of these materials, including a variety of concretes, rocks, bricks and others are non-linear deformation, the limit difference in stress-strain strength and weak signals of small amplitude in the transmitted pulse, which characterize the response of material and which must be registered with strain gages pasted on a measured bar.

\section{Uniaxial compression}

Among known up to date methods of dynamic testing, the Kolsky method [9] based on the Split-Hopkinson Pressure Bar (SHPB) has been widely used. The traditional technique allows testing a wide range of materials under compression in the strain rate range $10^{2} \div 10^{4} \mathrm{c}^{-1}$ (Fig. 1). The main advantages of the SHPB method are the simplicity of realization, the correct theoretical basis of the phenomena, occurring in the system of two thin long elastic bars and a short elastoplastic specimen between them, the exact determination of significant (tens of percent) deformations of the specimen, due to indirectness of the measurements and insignificant inertia of strain gages, recording the deformation pulses in bars, as well as the exclusion of the specimen bending due to its short length. In addition, this method allows to record the change in the strain rate history during the whole deformation process of the specimen.

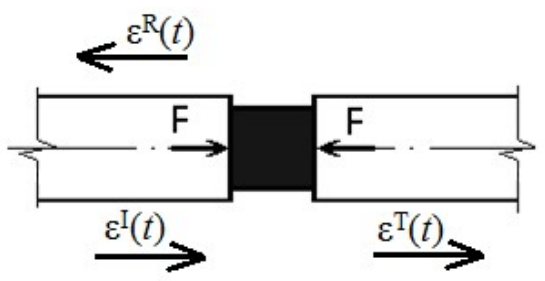

Fig. 1. Specimen in the system of split bars under compression testing

The Kolsky method is based on certain prerequisites and assumptions. Firstly, the duration of a loading pulse is much longer than the time of the wave passage along the specimen length due to its small length, that leads to multiple waves reflection from the specimen ends and, consequently, a uniaxial stress state with a uniform stress strain distribution along the specimen length is realized; thus, despite the high stress-strain rate of the specimen, the test can be regarded as quasi-static. Secondly, the elastic limit of the measuring bars must be considerably higher than the yield limit of the specimen. Thirdly, there is no dispersion when the waves propagate in dimensional bars. Finally, the distribution of the strain profile within the cross-section of the bar is uniform, and transverse vibrations of bar particles are absent.

The stress, strain and strain rate in the specimen are calculated from the pulse deformation, recorded in the sections of loading and supporting measuring bars as:

$$
\sigma_{\text {compression }}(t)=\frac{E A}{A_{0}} \cdot \varepsilon^{T}(t)
$$




$$
\begin{aligned}
& \varepsilon_{\text {compression }}(t)=-\frac{2 C}{L_{0}} \int_{0}^{t} \varepsilon^{R}(t) \cdot d t \\
& \dot{\varepsilon}_{\text {compression }}(t)=-\frac{2 C}{L_{0}} \cdot \varepsilon^{R}(t)
\end{aligned}
$$

where $E, A, c$ are the modulus of elasticity, the cross-sectional area, and the sound velocity in the measuring bars, respectively; $A_{0}, L_{0}$ are the cross-sectional area and the length of the specimen, respectively; $\varepsilon^{R}(t), \varepsilon^{T}(t)$ are the deformation pulses reflected from the specimen and transmitted through it, respectively, registered in the sections of the loading and supporting measuring bars, respectively.

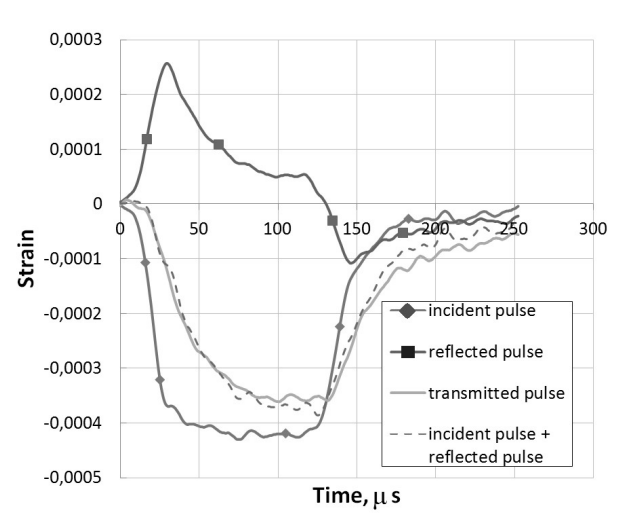

a

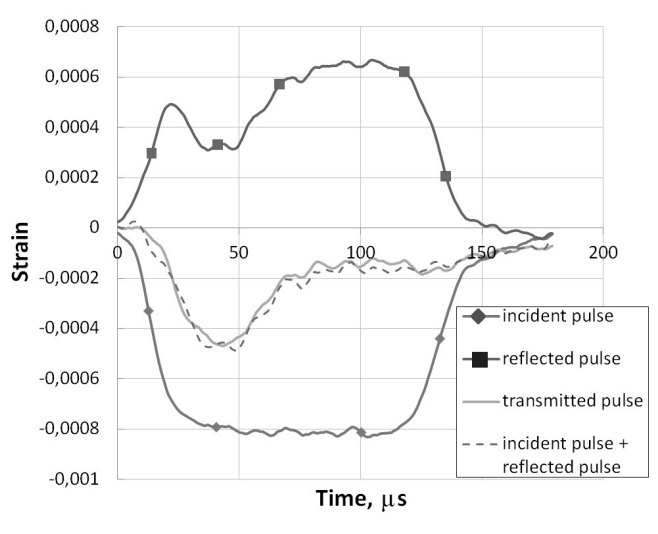

b

Fig. 2. Strain pulsed registered at cross-sections of the measuring bars when the specimen of ceramic brick maintains its integrity (a) and when it totally disintegrates (b)

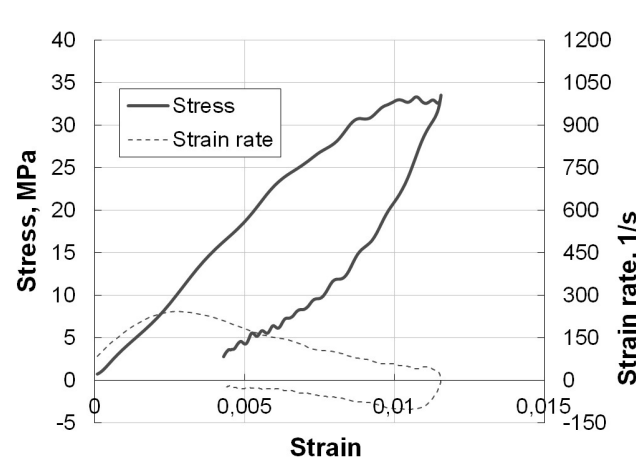

a

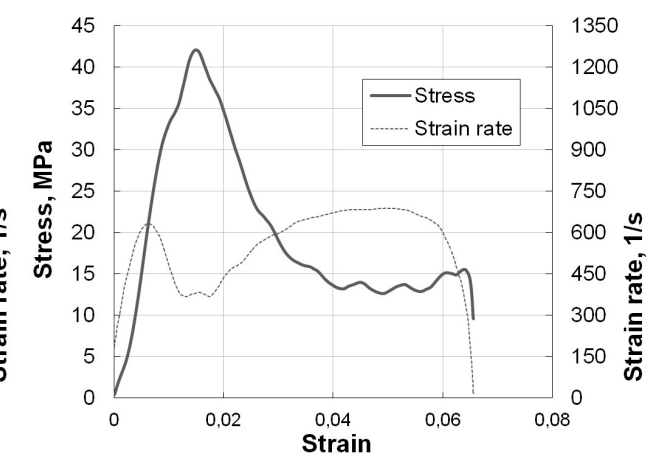

$\mathrm{b}$

Fig. 3. The average diagrams of the deformation of ceramic brick when the specimen maintains its integrity (a) and when it totally disintegrates (b)

Some recommendations for the testing of brittle materials are given in [10-12]. According to them, the length to diameter ratio of the specimen should be $0.3-1$. To reduce friction effect in the process of radial distribution of the specimen, it is necessary to use the lubrication of the contact surfaces of the bars and the specimen. Equality of forces 
acting on the sample during its deformation is evaluated on the basis of time-synchronized deformation pulses of measuring bars. For example, Fig. 2 shows the time synchronized deformation pulses of measuring bars obtained during the testing of ceramic bricks, in case the specimen retains its integrity (Fig. 2a) and if it is completely failed (Fig. 2b). It can be noted that in the absence of specimen failure, the decay of the amplitude of a transmitted pulse occurs after the end of the action of an incident pulse, and also a «negative tail» is observed in a reflected pulse, which reduces deformation and creates an unloading branch on the material deformation diagram. In case of the specimen failure in the first loading cycle, the amplitude of the transmitted pulse sharply decreases and the amplitude of the reflected pulse increases simultaneously up to the value of the amplitude of an incident wave, which continues its effect. In order to detect a transmitted small amplitude pulse, semiconductor strain gauges with higher sensitivity as compared with foil ones are employed on the supporting bar. The average diagrams of the deformation of the samples corresponding to the described loading regimes are shown in Fig. 3.

This technique allows to obtain dynamic deformation diagrams in stress-strain or stresstime axes at different strain rates of experimental specimen. On the basis of these diagrams, the maximum stress, ultimate deformation and the time before failure are determined, corresponding to this stress under different loading modes. In addition, by integrating the stress-strain curves obtained in the axes, it is possible to calculate the value of the specific energy necessary for deformation and subsequent specimen failure.

\section{Splitting}

To obtain properties under dynamic stretching, a modification of the method is used - a splitting test or the «Brazilian test» (Fig. 4). The dependence of the tensile stress on time for a cylindrical specimen is constructed on the basis of the deformation pulse recorded in the section of the supporting measuring bar as:

$$
\sigma_{\text {tension }}(t)=\frac{2 E A}{\pi L_{0} D_{0}} \cdot \varepsilon^{T}(t)
$$

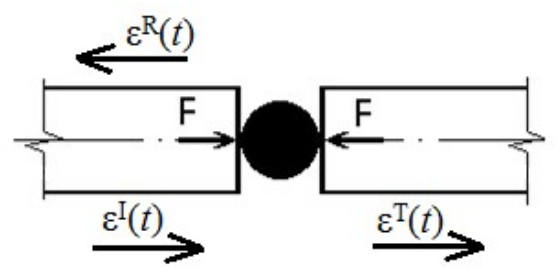

Fig. 4. The sample in the system of splitting bars at the splitting test

The applicability analysis of this technique was carried out in [8], where it is noted that the «Brazilian test» can be used to determine the tensile strength of brittle materials, when the behavior of material is close to elastic and the equilibrium loading state of the specimen is achieved, and its failure occurs along the diametrical plane. The profiles of time-synchronized deformation pulses of measuring bars, obtained during testing of ceramic bricks, showing the practical forces equality acting on the surfaces ends of the specimen, which causes the state of its equilibrium loading are shown for example in Fig. 5. The average deformation diagrams of ceramic brick constructed for different velocities of the striker are shown in Fig. 6. The type of ceramic brick destroyed under splitting is shown in Fig. 7. 


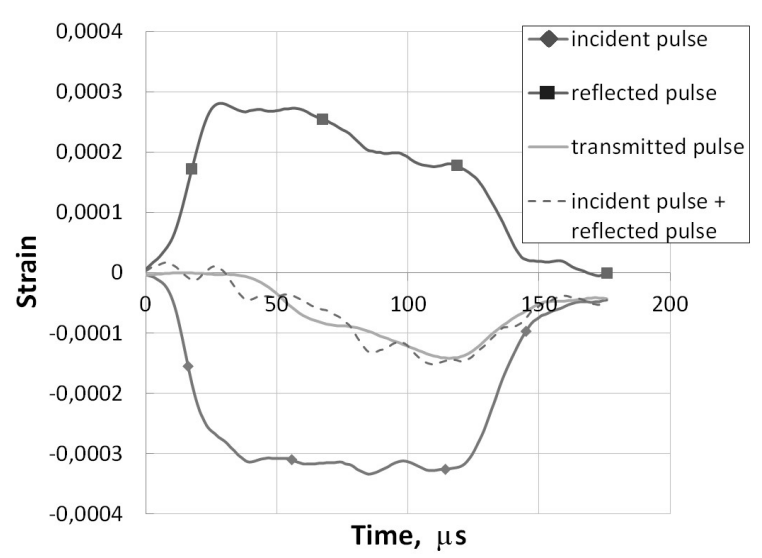

Fig. 5. Strain pulses registered at cross-sections of the measuring bars at testing specimen of ceramic brick on the splitting

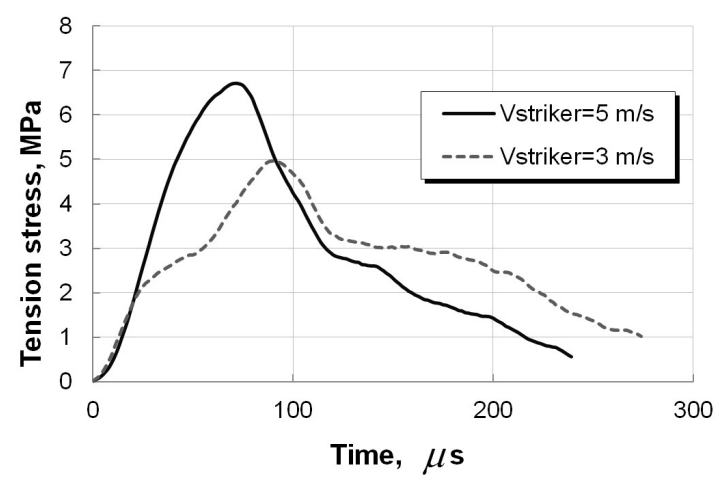

Fig. 6. Average deformation diagram of ceramic brick at splitting.

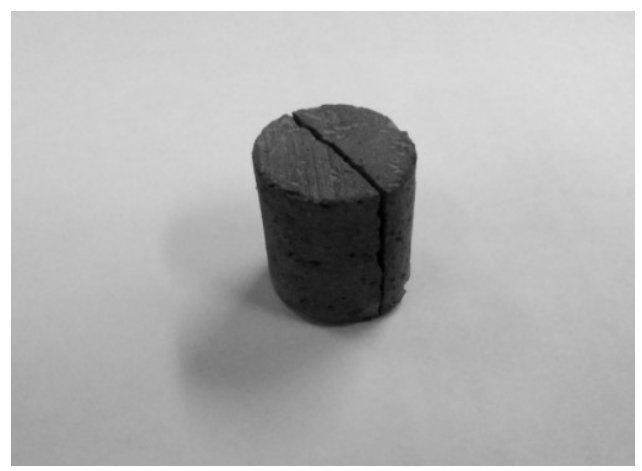

Fig. 7. The sample of a ceramic brick after splitting test

\section{Indirect tensile}

To determine the mechanical characteristics of brittle materials under tensile, a modification of the Kolsky method [7] was proposed and implemented, based on the fact that the strength of brittle media under compression is higher than their tensile strength (Fig. 8). The test circuit is similar to the compression version, but the loading bar must be at least twice as long as the supporting bar, the latter has a free rear end and the specimen is 
connected to the bars by means of an adhesive. Longitudinal compression pulse is applied to the loaded end of the first measuring bar and excites in it an elastic one-dimensional compression wave $\varepsilon_{11}(t)$. The amplitude of this wave must be such that it does not cause damage to the specimen (that is, under compression the specimen is deformed elastically). Then the initial pulse almost completely passes through the specimen into the second bar by the wave $\varepsilon_{12}(t)$ and, reaching the free end of this bar, is reflected in the form of a tensile wave. This tensile pulse $\varepsilon_{12}=-\varepsilon^{\mathrm{I}}(t)$ is the initial incident wave for the break of the sample. From the moment of formation of the tensile pulse from the free end of the bar and its propagation in the opposite direction along the bar, the experimental scheme is similar to the compression test scheme. Reaching the sample, the tensile pulse $\varepsilon^{\mathrm{I}}(t)$ partially passes through it to the first bar by the wave $\varepsilon^{\mathrm{T}}(t)$, is partially reflected back to the second bar by the wave $\varepsilon^{\mathrm{R}}(t)$. If the tensile pulse amplitude $\varepsilon^{\mathrm{I}}(t)$ is higher than the tensile strength of the specimen, then it causes its failure. Dependences of stress, strain and strain rate in the specimen on time are determined by the traditional formulas of the SHPB method. Timesynchronized deformation pulses recorded in the tensile cycle of the specimen of fine-grain concrete, proving the equality of forces at the ends of the specimen and indicating the uniformity of its stressed state are presented in Fig. 9. The deformation diagram obtained by testing fine-grained concrete using the described technique is shown in Fig. 10. Fig. 11 shows the type of failure of the specimen of fine-grain concrete after tensile wave loading.

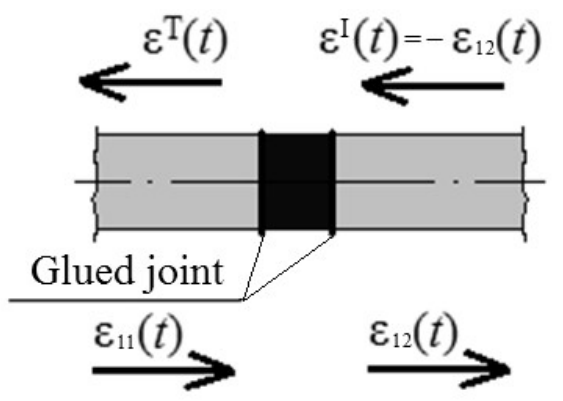

Fig. 8. The specimen in the system of split bars at indirect tensile testing

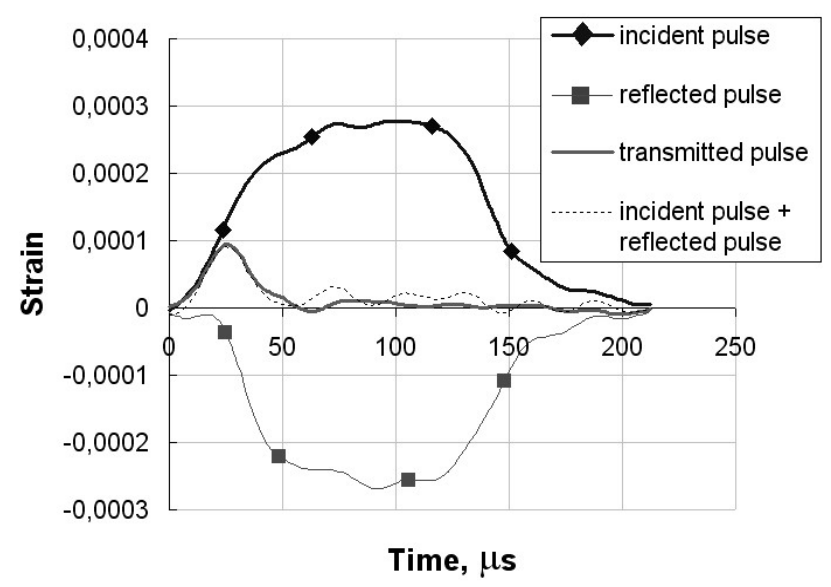

Fig. 9. Deformation pulses recorded in cross-sections of measuring bar at straight tensile of fine-grain concrete 


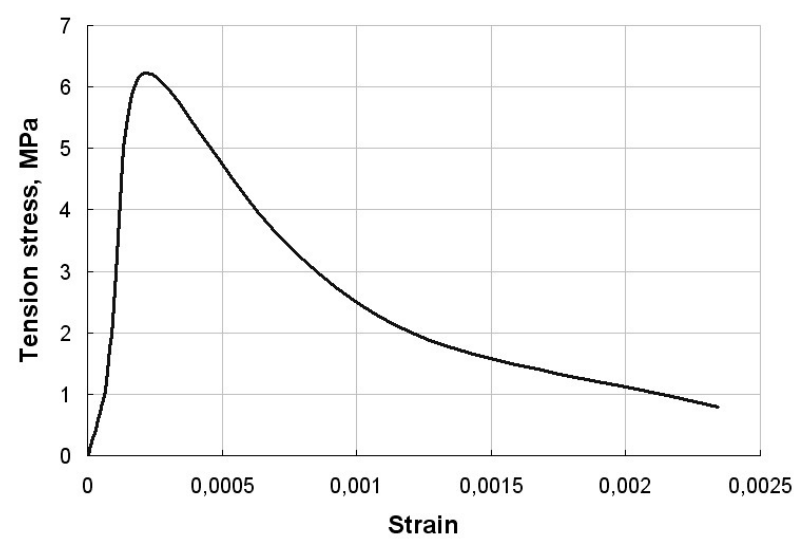

Fig. 10. Deformation diagram of fine-grain concrete obtained at indirect tensile

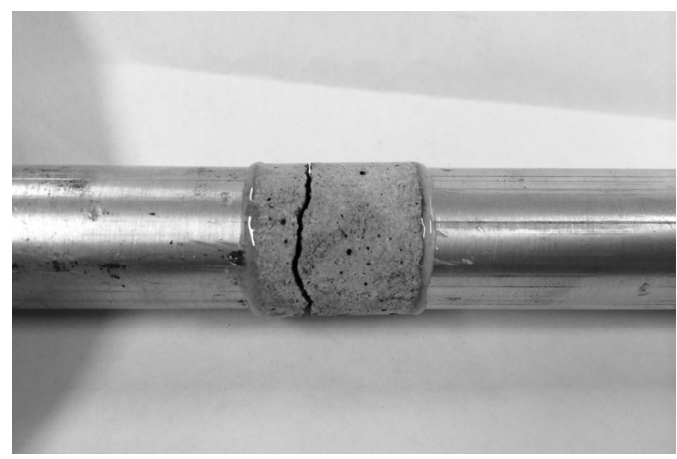

Fig. 11. A specimen of fine-grain concrete after a direct tensile test

\section{Shear}

A shear in its pure form is the division of an element into two parts along a section to which shear forces are applied. In proposed and implemented modification of the Kolsky method to determine the dynamic strength of brittle materials on a shear [6], the specimen of studied material is located in a rigid jacket cut at an angle $\alpha$ to the horizontal (Fig. 12). In order for the specimen to be subjected to only a section without compression, its length must be less than the length of the split jacket. The jacket with the specimen is placed between the measuring bars: loading and supporting. After the impact of the striker on the loading bar, a slight displacement of two parts of the split jacket relatively to the shear plane takes place. Since brittle materials have low deformability, this causes deformation and failure of the specimen. The shear stress dependence on time in the specimen is determined from the deformation pulse recorded in the supporting bar.

$$
\tau(t)=\frac{E A \cos \alpha \sin \alpha}{\pi R^{2}} \cdot \varepsilon^{T}(t)
$$

where $\mathrm{R}$ is an inner jacket radius.

As an example, in Fig. 13 shows the average dependence of the shearing stress on time, obtained during tests of fine-grain fibrous concrete. The type of the specimen failure is shown in Fig. 14. 


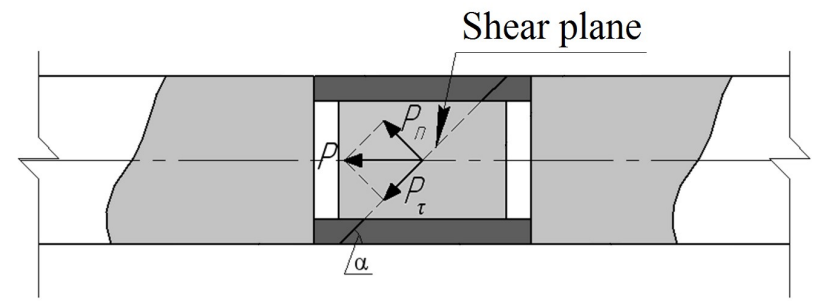

Fig. 12. Shear test scheme

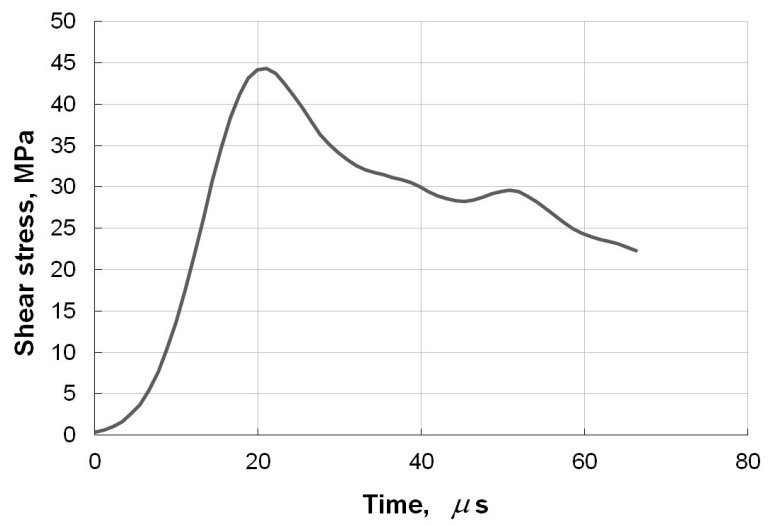

Fig. 13. Dynamic diagram obtained at shear test of fiber-reinforced concrete

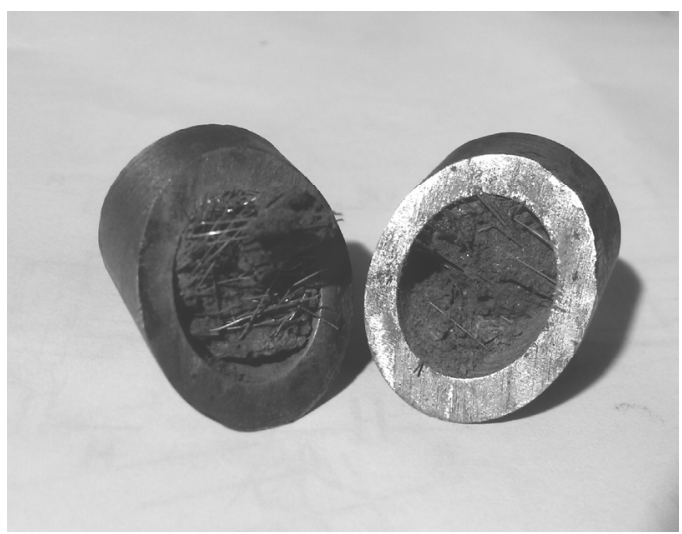

Fig. 14. Specimen of fiber-reinforced concrete after shear test

\section{Jacket compression}

In order to obtain the properties of brittle media under complex stress state, a modification of the Kolsky method for testing materials under passively confined radial strain was developed [2-3, 13]. In this case, a test specimen is located between the ends of the measuring bars in a rigid steel elastically deformable jacket that confines its radial distribution (Fig. 15). Under such loading conditions, it is possible to determine all the components of the stress and strain gauge at each point of the specimen:

$$
\sigma_{1}=\sigma_{x} ; \sigma_{2}=\sigma_{3}=\sigma_{r} ; \varepsilon_{1}=\varepsilon_{x} ; \varepsilon_{2}=\varepsilon_{3}=\varepsilon_{r}
$$

where $\sigma_{x}$ and $\varepsilon_{x}$ are longitudinal stress and strain, $\sigma_{r}$ and $\varepsilon_{r}$ are radial stress and strain in the specimen. 


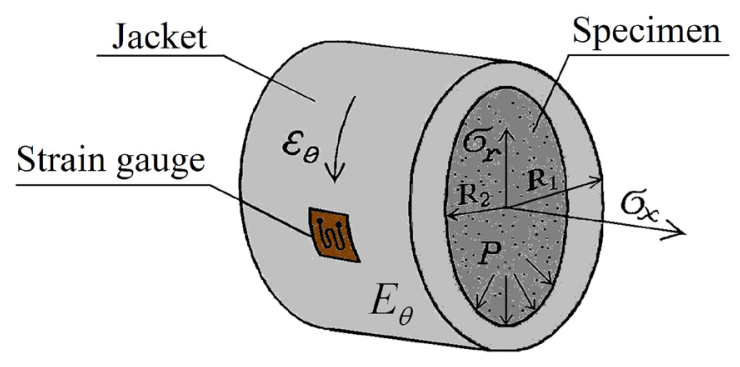

Fig. 15. Specimen scheme in a jacket

Longitudinal stresses, strains and longitudinal deformation rate are determined by the formulas of the traditional Kolsky method, whereas the radial stresses and strains are determined by the deformation pulse recorded on the steel jacket $\varepsilon_{\theta}(t)$ from the formulas obtained from the solution of the Lame problem on the elastic deformation of a thickwalled pipe (or hollow tube cylinder) under internal pressure:

$$
\begin{aligned}
& \sigma_{r}(t)=\frac{1}{2 R_{2}^{2}}\left[E_{\theta}\left(R_{1}^{2}-R_{2}^{2}\right) \varepsilon_{\theta}(t)\right] \\
& \varepsilon_{r}(t)=\frac{R_{2}^{2}(1-v)+R_{1}^{2}(1+v)}{2 R_{2}^{2}} \cdot \varepsilon_{\theta}(t)
\end{aligned}
$$

where $\mathrm{E}_{\theta}, v$ are elasticity modulus and the Poisson's ratio of jacket material, respectively; $\mathrm{R}_{1}$ and $\mathrm{R}_{2}$ are outer and inner jacket radius, respectively.

The above technique makes it possible to calculate a wide range of properties of the test material: shear resistance, lateral pressure coefficient, specimen pressure, volumetric deformation, stress intensity and strain intensity. Therefore, apart from obtaining a diagram of uniaxial specimen compression under passive confined radial strain, the used variant of the Kolsky method is employed to obtain the volume compressibility curve and the dependence of shear resistance on pressure.

As an example, Fig. 16 shows the average pressure dependences of the volume strain, as well as the average dependences of the equivalent stress (stress intensity) on pressure to the moment when the longitudinal and radial stresses reach their maximum values obtained at different longitudinal strain rates for fine-grain concrete. In the presented diagrams solid lines show the dependence of pressure versus the volume strain or stress intensity versus the pressure. Dashed lines show the dependence of the longitudinal strain rate versus the volume strain or longitudinal strain rate versus pressure. Markers (their absence) correspond to the same loading conditions.

\section{Conclusions}

On the basis of the Kolsky method, modifications have been developed and implemented that make it possible to carry out tests of brittle materials under tension, shear and a complex stress strain state. The described techniques make it possible to obtain a wide range of properties of brittle materials at dynamic strain rates. 

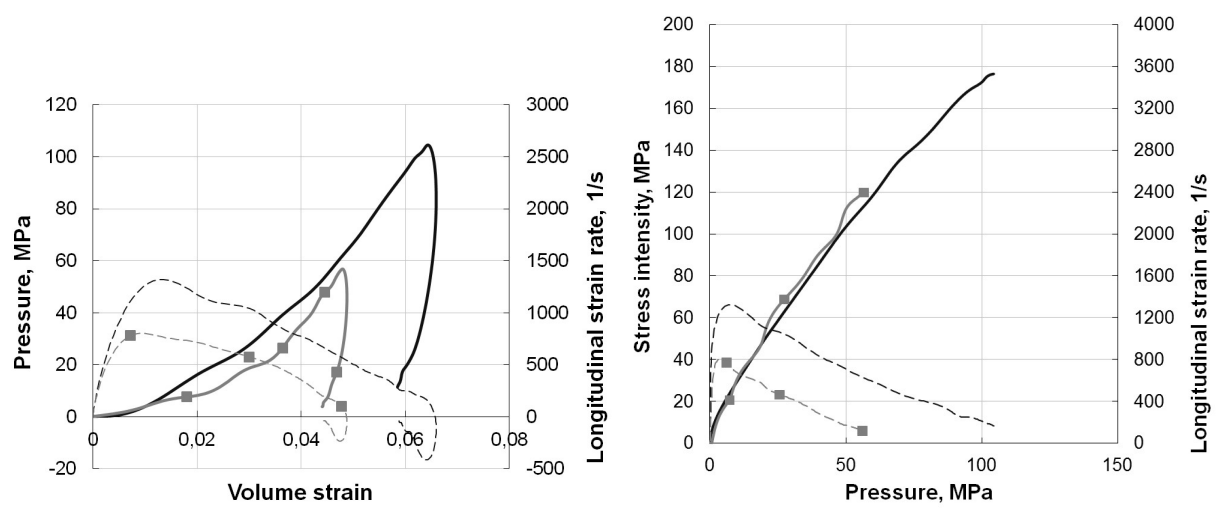

Fig. 16. Test results of fine-grain concrete on jacket compression

The research was carried out at the expense of the grant of the Russian Science Foundation (project No. 17-79-20161) regarding the development and approbation of test methods, as well as the state assignments of the Ministry of Education and Science of the Russian Federation (grant No. 9.6109.2017 / 6.7) regarding the study of material properties.

\section{References}

1. A.M. Bragov, A.K. Lomunov, International Journal of Impact Engineering 16(2), 321 (1995)

2. A.M. Bragov, G.M. Grushevsky, A.K. Lomunov, DYMAT Journal 1(3), 253 (1994)

3. A.M. Bragov, G.M. Grushevsky, A.K. Lomunov, Exp..Mech. 36(3), 237 (1996)

4. A.M. Bragov, A.K. Lomunov, I.V.Sergeichev, Journal of Applied Mechanics and Technical Physics 42(6), 1090 (2001)

5. A.M. Bragov, A.Yu. Konstantinov, A.K. Lomunov, Tech. Phys. Let., 34(5), 439 (2008)

6. A.M. Bragov, A.K. Lomunov, A.Yu. Konstantinov, D.A. Lamzin, Technical Physics Letters 43(1), 130 (2017)

7. A.M. Bragov, A.K. Lomunov, A.Yu. Konstantinov, D.A. Lamzin, Privolzhsky scientific journal, 2, 9 (2016) [in Russian]

8. T. Rodriguez, C. Navarro, V. Sanchez-Galvez, Journal de Physique IV 4, 101 (1994)

9. H. Kolsky, Proc. Phys. Soc., 62B (1949)

10. Q.M. Li, H. Meng, International Journal of Solids and Structures 40, 343 (2003)

11. M. Zhang, H.J. Wu, Q.M. Li, F.L. Huang, International Journal of Impact Engineering 36, 1327 (2009)

12. Q.M. Li, Y.B. Lu, H. Meng, International Journal of Impact Engineering 36, 1335 (2009)

13. A.M. Bragov, A.K. Lomunov, A.Yu. Konstantinov, D.A. Lamzin, V.V. Balandin, Journal Problems of Strength and Plasticity 78(4), 378 (2016) [in Russian] 